\title{
Review of: "Characterization of the Genetic Basis and Biological Significance of Heteroblasty in Soybean"
}

\author{
Hai Nian ${ }^{1}$ \\ 1 South China Agricultural University
}

Potential competing interests: The author(s) declared that no potential competing interests exist.

Leaf morphology is an important agronomic trait affecting the light energy utilization efficiency and being associated with soybean yield and quality. This manuscript reported heteroblasty in soybean, and detected the QTLs of 11 traits associated with leaf morphology, seed quality, and grain yield by using two recombinant inbred line (RIL) populations grown in two environments. The authors identified eight colocated QTLs for leaf morphology and seed quality and grain yield, which coincided with extensive leaf morphologies that contributed to seed quality and grain yield formation. Generally, this manuscript is well written and organized. Meanwhile, this study provided some novel information breeding high yield soybean cultivars with better quality through optimizing shoot architecture. Therefore, I suggested this manuscript could be accepted with some minor revision.

Comment:

1. There are some spelling error in the manuscript, such as:

2. In page 2, "89.6" would be "89.60".

3. In page 3, "rounder leaflets were are associated with enriched fruit sugar content" would be "rounder leaflets were associated with enriched fruit sugar content".

4. In page 6, "GFF3 files from the soyabean genome annotations" would be "GFF3 files from the soybean genome annotations".

5. In page 7, "3.3" would be "3.30".

6. In page 8, "absolute values of Kurtosis Skewness for all" would be "absolute values of Kurtosis and Skewness for all".

Please check the manuscript carefully.

1. What is the difference between heteroblasty and heterophylly? And Does the introduction of "heterophylly" on the second page need to be modified into "heteroblasty"? 